\title{
Excitation-contraction coupling of human induced pluripotent stem cell-derived cardiomyocytes
}

\author{
Christopher Kane, Liam Couch and Cesare M. N. Terracciano * \\ Laboratory of Cell Electrophysiology, National Heart and Lung Institute, Imperial College London, London, UK
}

Induced pluripotent stem cell-derived cardiomyocytes (iPSC-CMs) hold enormous potential in many fields of cardiovascular research. Overcoming many of the limitations of their embryonic counterparts, the application of iPSC-CMs ranges from facilitating investigation of familial cardiac disease and pharmacological toxicity screening to personalized medicine and autologous cardiac cell therapies. The main factor preventing the full realization of this potential is the limited maturity of iPSC-CMs, which

OPEN ACCESS

Edited by:

Cedric Viero,

Saarland University, Germany

Reviewed by:

Nina D. Ullrich,

University of Heidelberg, Germany

John James Mackrill,

University College Cork, Ireland

*Correspondence:

Cesare M. N. Terracciano, Laboratory of Cell Electrophysiology, Imperial Centre for Translational and Experimental Medicine, Hammersmith

Hospital, DuCane Road,

London W12 ONN, UK

c.terracciano@imperial.ac.uk

Specialty section:

This article was submitted to

Stem Cell Treatments,

a section of the journa

Frontiers in Cell and Developmental

Biology

Received: 26 May 2015 Accepted: 11 September 2015

Published: 29 September 2015

Citation:

Kane $C$, Couch $L$ and

Terracciano CMN (2015)

Excitation-contraction coupling of

human induced pluripotent stem

cell-derived cardiomyocytes.

Front. Cell Dev. Biol. 3:59.

doi: 10.3389/fcell.2015.00059 display a number of substantial differences in comparison to adult cardiomyocytes. Excitation-contraction (EC) coupling, a fundamental property of cardiomyocytes, is often described in iPSC-CMs as being more analogous to neonatal than adult cardiomyocytes. With $\mathrm{Ca}^{2+}$ handling linked, directly or indirectly, to almost all other properties of cardiomyocytes, a solid understanding of this process will be crucial to fully realizing the potential of this technology. Here, we discuss the implications of differences in EC coupling when considering the potential applications of human iPSC-CMs in a number of areas as well as detailing the current understanding of this fundamental process in these cells.

Keywords: induced pluripotent stem cell-derived cardiomyocytes, excitation-contraction coupling, stem cell maturation, disease modeling, pharmacological screening

\section{Introduction}

Induced pluripotent stem cells (iPSCs) were first described by Shinya Yamanaka and colleagues in 2006 who detailed the reprograming of adult mouse fibroblasts into a pluripotent state (Takahashi and Yamanaka, 2006). The means to generate de novo cardiomyocytes from iPSCs was then described in mice in 2008 (Mauritz et al., 2008; Narazaki et al., 2008) and later in human (Zhang et al., 2009). Since then, interest in induced pluripotent stem cell-derived cardiomyocytes (iPSC-CMs) has steadily increased. iPSC-CMs represented a step-change in stem cell research, surmounting ethical issues surrounding consent and the use of embryonic material that has restricted research using embryonic stem cells (ESCs), making them a much more attractive technology to expand into wider research and industry. Furthermore, as iPSCs and their derivatives are specific to the genetic makeup of the cell donor, whole avenues of research open up in terms of personalized medicine and familial disease, applications that could not have been considered with human ESC-CMs (hESC-CM).

Excitation-contraction (EC) coupling is not only one of the fundamental properties of cardiomyocytes, turning rhythmical electrical stimulation into the production of mechanical force, but is also central to determining many other electrophysiological and mechanical cell properties and is a key process that becomes dysregulated in almost all cardiac diseases. Studies of EC coupling 
in human iPSC-CMs (hiPSC-CMs) have described substantial differences between their properties and those of adult cardiomyocytes. While understanding these differences will be crucial to fully realizing the potential of hiPSC-CMs, research to date is limited. Furthermore, electrophysiological study of hiPSC-CMs is confounded by the variability of these cells in culture. We have previously shown the markedly increased variability in action potential morphology when hiPSC-CMs are cultured as single cells as compared to in a monolayer. As patch clamping studies necessitate the use of individual cells, these findings raise questions over the usefulness of investigations performed in this manner (Du et al., 2015).

In this mini-review we discuss the importance of EC coupling when considering the full potential of hiPSC-CMs in a wide range of fields, as well as detailing our current understanding of the components of this fundamental property of cardiomyocytes.

\section{Excitation-Contraction Coupling in}

\section{Drug Discovery and Screening}

The development of new pharmacological agents is a long and hugely expensive process, taking up to 10 years to bring a novel agent to market with an average cost of \$2.6 billion in 2014 (Mullard, 2014). In spite of the massive investment involved in this process, FDA approvals of "new chemical entities" are steadily declining (Paul et al., 2010). Up to $90 \%$ of compounds that pass pre-clinical screening fail at the highly expensive clinical trial level, with around one third showing unforeseen sideeffects (Kola and Landis, 2004). Cardiotoxicity alone accounts for $45 \%$ of post-approval withdrawal of compounds (Stevens and Baker, 2009). Improvements to pre-clinical screening of novel compounds for efficacy and toxicity would provide an enormous boon to the pharmaceutical industry, both in terms of reducing expensive attrition rates and improving safety.

The main weakness of pre-clinical screening is the use of in vitro and animal models that do not sufficiently reproduce human physiology and disease. As such hiPSC-CMs are a highly attractive option when considering replacements for current models. There has been much discussion about the suitability of hiPSC-CMs in this application (Khan et al., 2013; Sharma et al., 2013; Sinnecker et al., 2014), however there is still concern that hiPSC-CMs do not recapitulate adult human physiology enough to be useful (Jonsson et al., 2012). Furthermore, while industry utilizes a wide range of assays to assess drug effects, many rely directly or indirectly on the underlying process of EC coupling. An understanding of iPSC-CM Ca ${ }^{2+}$ handling is therefore crucial to properly interpret these experiments and consider their validity in the context of adult pharmacological therapy.

\section{Cell Therapy}

A further exciting application of hiPSC-CMs is in the realm of cell therapy, potentially providing an autologous cell source that could be used in the treatment of a wide range of pathologies. As has been discussed elsewhere (Chen et al., 2009), there are a number of substantial electrophysiological challenges that need to be overcome to successfully employ
hiPSC-CMs in cell therapy. The arrhythmogenic risks of using electrophysiologically immature cardiomyocytes in cell therapy has been demonstrated in detail (Zhang, 2002; Chang et al., 2006; Lalit et al., 2014; Smit and Coronel, 2014). As such a firm understanding of the EC coupling properties of these cells will be important in overcoming the risks and improving the benefits of cell therapy techniques utilizing hiPSC-CMs.

\section{Disease Modeling}

A third major area that has benefited from iPSC technology is that of disease modeling and in particular the modeling of inherited cardiac diseases. Since their discovery, iPSC-CM lines for a wide range of pathologies have been developed, such as channelopathies (Liu and Trudeau, 2015; Ma et al., 2015); cardiomyopathies (Caspi et al., 2013; Lin et al., 2015); as well as mutations in $\mathrm{Ca}^{2+}$ handling machinery such as ryanodine receptors and calsequestrin (Novak et al., 2012; Di Pasquale et al., 2013). Still, there is concern over the ability of these cells to truly recapitulate the diseases they model (Bellin et al., 2012; Blazeski et al., 2012; Rajamohan et al., 2013). Indeed investigators modeling arrhythmogenic right ventricular dysplasia using hiPSC-CMs described how the induction of more mature metabolic properties was essential to accurately model adult-onset cardiac disease (Wen et al., 2015). Therefore, understanding the current electrophysiological properties of hiPSC-CMs will be crucial for proper interpretation of investigations into diseases modeled in this way.

\section{Calcium Handling in Induced Pluripotent Stem Cell-derived Cardiomyocytes}

$\mathrm{Ca}^{2+}$-induced $\mathrm{Ca}^{2+}$ release (CICR) is the key link between electrophysiological stimulation and the production of mechanical force as well as a feature that distinguishes cardiomyocytes from other types of muscle cells. Cardiomyocyte depolarization causes $\mathrm{Ca}^{2+}$ entry via voltage-sensitive Ltype $\mathrm{Ca}^{2+}$ channels, triggering the release of $\mathrm{Ca}^{2+}$ from the sarcoplasmic reticulum (SR) via ryanodine receptors (RyR), raising $\left[\mathrm{Ca}^{2+}\right]_{i}$ and causing contraction. Relaxation is achieved by removing the $\mathrm{Ca}^{2+}$ from the cytosol, either by re-sequestering it in the SR via the sarco-endoplasmic reticulum ATP-ase (SERCA), or extruding it into the extracellular space through the $\mathrm{Na}^{+}-\mathrm{Ca}^{2+}$ exchanger (NCX) or $\mathrm{Ca}^{2+}$ ATPase pump (Bers, 2002; Figure 1).

While hiPSC-CMs show robust $\mathrm{Ca}^{2+}$ transients, they rise and decline at a substantially slower rate (Lee et al., 2011; Hwang et al., 2015). The rate of decline of $\mathrm{Ca}^{2+}$ transients assessed in a number of iPSC-CM lines showed $\kappa$-values of $0.84-1.15 \mathrm{~s}^{-1}$ compared to 2.41 and $5.75 \mathrm{~s}^{-1}$ in adult rabbit and mouse cardiomyocytes respectively (Hwang et al., 2015). Exhibiting a strong adrenergic response (Yokoo et al., 2009; Germanguz et al., 2011), hiPSC-CMs display a negative force-frequency response (FFR; Germanguz et al., 2011), suggesting reduced basal SERCA function and an inability to utilize more of their $\mathrm{Ca}^{2+}$ stores. 

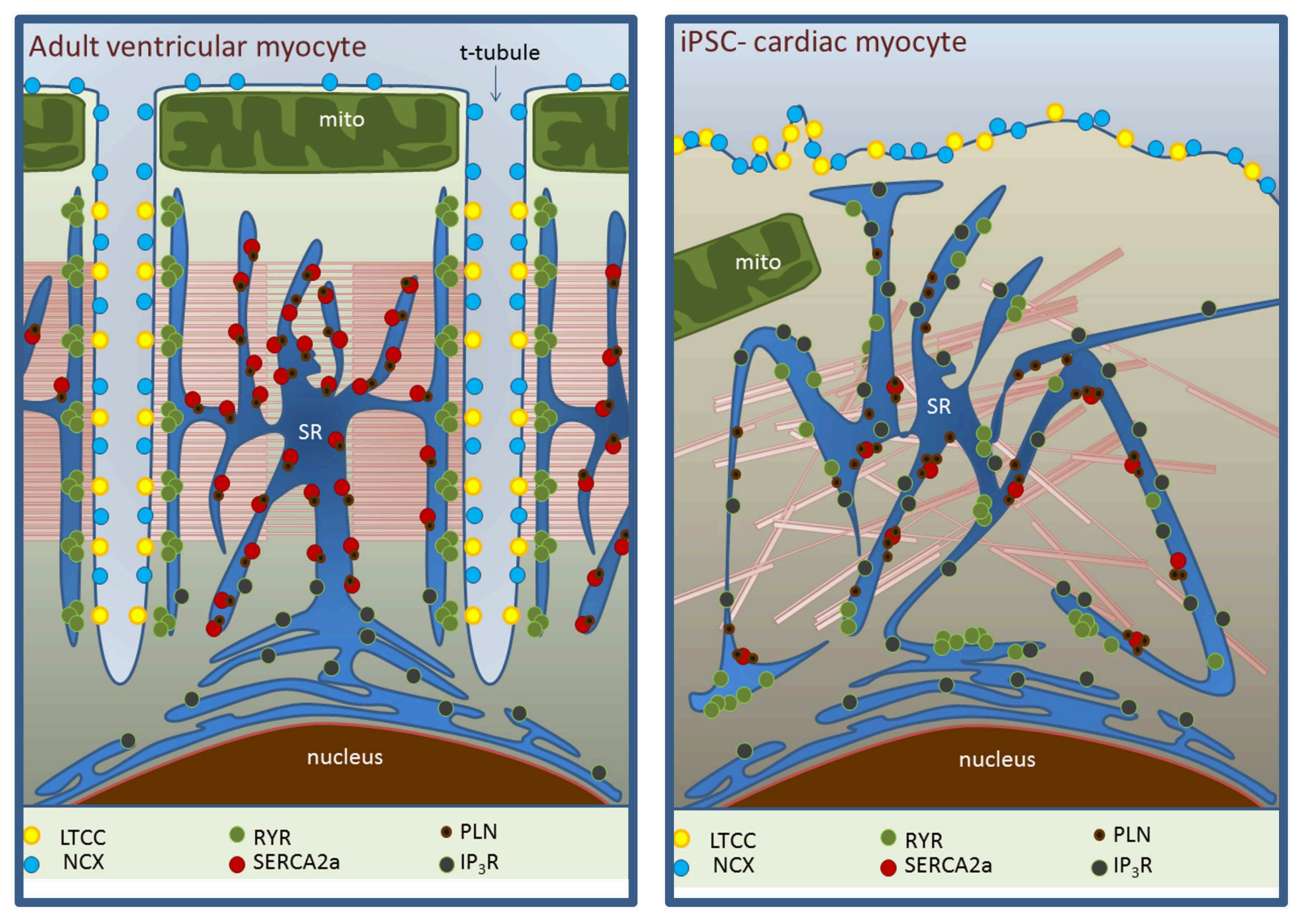

FIGURE 1 | Ultrastructural differences between adult myocytes (left) and iPSC-CMs (right) in EC coupling domains. The absence of t-tubules in iPSC-CM is associated with the lack of regular organization of L-type $\mathrm{Ca}^{2+}$ channel (LTCC)-RyR complexes and less homogeneous distribution of RyRs. SERCA expression is reduced with a maintained phospholamban (PLN) expression. NCX expression may be maintained but its ability to extrude $\mathrm{Ca}^{2+}$ in diastole is decreased (see main text). Inositol-triphosphate receptor $\left(\mathrm{IP}_{3} \mathrm{R}\right)$ activity is substantially higher.

\section{L-type $\mathrm{Ca}^{2+}$ Channels}

L-type $\mathrm{Ca}^{2+}$ channels are a vital component of EC coupling in hiPSC-CMs. We have previously shown that $\mathrm{CaV}_{1.2}$, the $\alpha-1 \mathrm{C}$ subunit of L-type $\mathrm{Ca}^{2+}$ channels, demonstrates similar expression levels to the adult human heart (Rao et al., 2013). Application of the $\mathrm{Ca}^{2+}$ channel blocker nifedipine and removal of $\left[\mathrm{Ca}^{2+}\right]_{0}$ both completely suppress whole cell $\mathrm{Ca}^{2+}$ transients in these cells, demonstrating that external $\mathrm{Ca}^{2+}$ is crucial for triggering SR $\mathrm{Ca}^{2+}$ release (Itzhaki et al., 2011). A number of studies investigating $\mathrm{Ca}^{2+}$ current $\left(\mathrm{I}_{C a}\right)$ through patch clamp techniques have measured substantial peak current densities, ranging from $-8 \mathrm{pA} / \mathrm{pF}$ (Zhang et al., 2013b) to $-17.1 \pm 1.7$ $\mathrm{pA} / \mathrm{pF}$ at RT (Ma et al., 2011), large in comparison to H1 hESCCMs (Sartiani et al., 2007), although similar to mouse cardiac myocytes (Hwang et al., 2015).

hiPSC-CMs respond to a wide range of $\mathrm{Ca}^{2+}$ channel antagonists in a similar manner to both H7 hESC-CMs and guinea pig myocytes (Kang et al., 2012). However, hiPSC-CMs display unusual responses to $\mathrm{Ca}^{2+}$ channel agonists. Bay K8644 has little effect on current amplitude (Kang et al., 2012; Ji et al., 2014), while responses to the $\mathrm{Ca}^{2+}$ channel activator FPL 64176 produced an increase in current in some cells but a reduction in others (Kang et al., 2012). Hyperphosphorylation of channels in heart failure is known to reduce the effect of Bay K8644 (Chen et al., 2008) and may explain these unusual effects in hiPSC-CMs. Understanding differences in channel pharmacology is crucial for the use of hiPSC-CMs in drug screening platforms.

\section{The Sarcoplasmic Reticulum}

\section{Ryanodine Receptors}

High conductance $\mathrm{Ca}^{2+}$ sensitive channels in the SR membrane, ryanodine receptors (RyRs) are the $\mathrm{Ca}^{2+}$ release mechanism of the SR, and are closely apposed to L-type $\mathrm{Ca}^{2+}$ channels. RYR2, the cardiac isoform of RyRs, appear diffuse throughout the cytosol on immunostaining with reports of both intense, punctate perinuclear (Itzhaki et al., 2011) and sub-sarcolemmal (Zhang et al., 2013b) staining. Expression is significantly lower in hiPSC-CMs than in H7 and HES3 hESC-CMs (Lee et al., 2011) and the adult human heart as well as levels of its regulatory proteins calsequestrin and triadin (Rao et al., 2013).

RyRs in hiPSC-CMs exhibit rapid, large releases of $\mathrm{Ca}^{2+}$ in response to the agonist caffeine (Fatima et al., 2011; Itzhaki et al., 2011; Lee et al., 2011; Kim et al., 2015; Zhang et al., 2015), and antagonism with ryanodine reduces whole cell $\mathrm{Ca}^{2+}$ transient amplitude. SR $\mathrm{Ca}^{2+}$ content, assessed by caffeine application, is similar to that found in adult rabbit cells (Hwang et al., 2015).

Activation of RyRs by $\mathrm{Ca}^{2+}$ entering the cell through $\mathrm{L}-$ type $\mathrm{Ca}^{2+}$ channels is crucial to the generation of mature calcium transients and there is evidence for functional CICR in hiPSC-CMs. hiPSC-CM transient amplitude reduced in a dose dependent manner in response to treatment with the selective L-type $\mathrm{Ca}^{2+}$ channel blocker nifedipine, abolishing transients altogether at $1 \mu \mathrm{M}$ (Itzhaki et al., 2011). Further, $\mathrm{Ca}^{2+}$ transients in these cells appear to be voltage dependent, with transient amplitude correlating positively with the size of the 
depolarization step used to activate $\mathrm{I}_{\mathrm{Ca}}$. Transients were elicited regardless of the direction of voltage change, consistent with $\mathrm{I}_{\mathrm{Ca}}$ activation on depolarization and re-activation of $\mathrm{I}_{\mathrm{Ca}}$ channels on repolarization. This dependence on $\mathrm{I}_{\mathrm{Ca}}$ rather than the direction of voltage change distinguishes cardiac CICR from voltageinduced $\mathrm{Ca}^{2+}$ release particular to skeletal muscle (Zhang et al., 2013b).

The initial rise in $\left[\mathrm{Ca}^{2+}\right]_{i}$ in $\mathrm{I}_{C a}$-induced $\mathrm{Ca}^{2+}$ release has been observed to occur at the sarcolemma before spreading inwards, suggesting inefficient CICR, with more internal, noncoupled RyRs being activated by the subsequent rise in $\left[\mathrm{Ca}^{2+}\right]_{i}$ as opposed to direct activation by $\mathrm{Ca}^{2+}$ channels (Lee et al., 2011; Zhang et al., 2013b). While a detailed EM study of iPSCCM ultrastructure identified some apposition of the SR with the sarcolemma (Gherghiceanu et al., 2011), hiPSC-CMs exhibit poor co-localization between $\mathrm{CaV}_{1.2}$ and RYR2 (Rao et al., 2013).

$\mathrm{Ca}^{2+}$ sparks are small releases of $\mathrm{Ca}^{2+}$ from the SR generated by the stochastic opening of small clusters of RyRs and represent the fundamental release unit of the CICR. In keeping with the spatial variability noted with global $\mathrm{Ca}^{2+}$ transients, $\mathrm{Ca}^{2+}$ sparks were observed to occur repeatedly at the same sites within the cell, with their properties differing between those occurring at the center vs. the periphery of the cell (Zhang et al., 2013a), suggesting an inhomogeneous clustering of RyR channels, as seen on immunofluoresence. The majority of sparks demonstrated multiple peaks in three-dimensional and temporal profiles, suggesting that more than one RyR cluster may be involved in each release event. Spark characteristics were comparable with those of adult rat cardiomyocytes.

\section{SERCA and Phospholamban}

SERCA2a, the cardiac isoform of SERCA, is expressed in hiPSCCMs at significantly lower levels compared to both hESC-derived myocytes (H7 and HES3 lines; Itzhaki et al., 2011; Lee et al., 2011) and the adult human heart (Rao et al., 2013).

Functionally, the rate of SR $\mathrm{Ca}^{2+}$ uptake is substantially slower in hiPSC-CMs than in adult rabbit and mouse cardiomyocytes (0.49-0.72 vs. 1.52 and $5.12 \mathrm{~s}^{-1}$ at RT; Hwang et al., 2015). Phospholamban is a key regulator of SR function, inhibiting SERCA activity when in its unphosphorylated form. Interestingly PLN expression is comparable to adult human cardiomyocytes (Rao et al., 2013); a high PLN:SERCA ratio may explain the low basal activity (Germanguz et al., 2011), and the concurrent ability of hiPSC-CMs to mount a strong response to adrenergic stimulation (Yokoo et al., 2009; Germanguz et al., 2011).

\section{Inositol-triphosphate Receptors}

$\mathrm{IP}_{3} \mathrm{R}$ antagonists 2 -APB and $\mathrm{U} 73122$ produced a significant reduction in transient amplitude in hiPSC-CMs (Itzhaki et al., 2011). Prominent $\mathrm{IP}_{3}$ activity is a common feature of neonatal (Poindexter et al., 2001) and failing adult human cardiomyocytes (Go et al., 1995; Ai et al., 2005). However, maximal application of U73122 completely inhibited $\mathrm{Ca}^{2+}$ transients, somewhat in conflict with the identical observation in the same study with high concentration thapsigargin, an inhibitor of SERCA. It has been suggested that U73122 has off target effects on SR
$\mathrm{Ca}^{2+}$ uptake (Macmillan and McCarron, 2010) but there is also evidence to support a complex role for $\mathrm{IP}_{3}$-mediated $\mathrm{Ca}^{2+}$ release in stem cell-derived $\mathrm{Ca}^{2+}$ handling. In mouse CMV ESC-CMs, inhibition of $\mathrm{IP}_{3}$ production with $\mathrm{U} 73122$ was associated with a decrease in spontaneous activity and $\mathrm{Ca}^{2+}$ spark frequency, while stimulation with ET-1 had positive chronotropic effects and increased diastolic $\left[\mathrm{Ca}^{2+}\right]_{i}$ in the presence of tetracaine (Kapur and Banach, 2007). Whilst it is unclear exactly how $\mathrm{IP}_{3}$-mediated $\mathrm{Ca}^{2+}$ release works together with other $\mathrm{Ca}^{2+}$ handling pathways, future work will likely further highlight the importance of this mechanism in iPSC-CM Ca ${ }^{2+}$ cycling.

\section{The Sodium-Calcium Exchanger NCX}

Expressed as the NCX1 isoform in the heart, NCX has a key role in maintaining $\mathrm{Ca}^{2+}$ homeostasis, moving $\mathrm{Ca}^{2+}$ out across the sarcolemma during systole in exchange for $\mathrm{Na}^{+}$moving into the cell at a ratio of $3: 1\left(3 \mathrm{Na}^{+}\right.$ions in for each $\mathrm{Ca}^{2+}$ ion out). Due to this stoichiometry, NCX generates a substantial inward current $\left(\mathrm{I}_{N C X}\right)$ that contributes to cell depolarization, pace maker activity and action potential duration (Blaustein and Lederer, 1999). Linking $\mathrm{Ca}^{2+}$ cycling and cell membrane potential, NCX is one key factor in afterdepolarization-based arrhythmogenesis and is as such of great interest as both a therapeutic target and a source of pharmacological toxicity (Pott et al., 2011).

Expression of NCX in hiPSC-CMs is reduced compared to two ESC-derived lines of cardiomyocytes (H7 and HES3; Lee et al., 2011), although more recently we have shown expression comparable to that in the adult human heart (Rao et al., 2013). NCX currents generated on application of $2 \mathrm{mM} \mathrm{Ca}^{2+}$ showed peak densities of $4.5 \pm 0.5 \mathrm{pA} / \mathrm{pF}$ at $37^{\circ} \mathrm{C}$ compared to an average of $<0.5 \mathrm{pA} / \mathrm{pF}$ in neonatal rat cardiomyocytes (Fine et al., 2013). A similar effect was seen in peak $\mathrm{I}_{N C X}$ elicited on application of $10 \mathrm{mM}$ caffeine, with peak densities of $\sim 2.6 \mathrm{pA} / \mathrm{pF}$ in hiPSCCMs at $24^{\circ} \mathrm{C}$ vs. $\sim 0.8 \mathrm{pA} / \mathrm{pF}$ in neonatal rat cardiomyocytes (Zhang et al., 2015), correlating with reports in additional investigations of $\sim 2.2 \mathrm{pA} / \mathrm{pF}$ in hiPSC-CMs (Zhang et al., 2013b). KB-R7943 reduced caffeine-induced current amplitude (Zhang et al., 2015), demonstrating the ability of the exchanger's forward mode to influence resting membrane potential. To date there has been no assessment of $\left[\mathrm{Na}^{+}\right]_{i}$ or the reversal potential of the exchanger in hiPSC-CMs and as such the possibility of substantial $\mathrm{Ca}^{2+}$ influx via reverse mode NCX remains unexplored.

Regarding the relative contribution of the two main mechanisms, SR $\mathrm{Ca}^{2+}$ uptake and NCX, to $\mathrm{Ca}^{2+}$ extrusion, Hwang et al. have recently reported that this is similar to adult rabbit myocytes, with approximately $60 \%$ of the $\mathrm{Ca}^{2+}$ released by caffeine removed by the SR and $40 \%$ by NCX, which may suggest robust and adequate diastolic function (Hwang et al., 2015). However, as previously discussed, the rate of decline of $\mathrm{Ca}^{2+}$ transients is extremely slow in hiPSCCMs with both absolute values for $\mathrm{SR} \mathrm{Ca}^{2+}$ uptake and NCXmediated $\mathrm{Ca}^{2+}$ extrusion significantly reduced. This also stands in contrast to the consistent observation that hiPSC-CMs display a negative FFR, a phenomenon observed in the failing heart, consistent with substantially reduced SR function. The similarity 
in relative contribution to $\mathrm{Ca}^{2+}$ extrusion may be peculiar to the experimental conditions used (room temperature, $0.5 \mathrm{~Hz}$ stimulation rate) and as such need to be interpreted with caution. In summary, hiPSC-CMs display significantly impaired diastolic $\mathrm{Ca}^{2+}$ removal.

\section{Spontaneous SR $\mathrm{Ca}^{2+}$ Release Events and NCX}

A key feature of hiPSC-CMs is that they beat spontaneously, making for a convenient means to confirm successful differentiation of stem cells into cardiomyocytes. Unexpectedly, study of this phenomenon has given us a number of insights into the regulation of $\mathrm{Ca}^{2+}$ handling in these cells.

Optical techniques assessing spontaneous $\mathrm{SR}^{2} \mathrm{Ca}^{2+}$ release identified small $\mathrm{Ca}^{2+}$ wavelets propagating within the cell, the majority of which were too small to trigger a global $\mathrm{Ca}^{2+}$ release (Kim et al., 2015). Successive wavelets appeared to originate repeatedly from the same sites throughout the cytosol, an observation also made above when looking at $\mathrm{Ca}^{2+}$ sparks, which increased in amplitude to a point where a global $\mathrm{Ca}^{2+}$ release was triggered.

Beating rate of the cells correlated positively with the application of isoproterenol and negatively with reduced $\left[\mathrm{Ca}^{2+}\right]_{o}$, as did the incidence of $\mathrm{Ca}^{2+}$ wavelets (Kim et al., 2015). The RyR stabilizer K201 (Kim et al., 2015), inhibitors tetracaine and ryanodine (Kim et al., 2015; Zhang et al., 2015), the NCX inhibitor SEA0400 (Kim et al., 2015) and SERCA2a inhibitor thapsigargin (Zhang et al., 2015) all reduced or abolished spontaneous activity in hiPSC-CMs, confirming that automaticity in these cells was dependent on spontaneous SR release generating a depolarizing current via NCX. Further, a combination of switch-clamp recording of membrane potential and GCamP6-FKBP, a $\mathrm{Ca}^{2+}$-sensing probe targeted at RyRs, showed that $\mathrm{Ca}^{2+}$ release events correlated with diastolic depolarization preceding the action potential upstroke (Zhang et al., 2015).

\section{Mitochondrial $\mathrm{Ca}^{2+}$}

The topic of mitochondrial $\mathrm{Ca}^{2+}$ in cardiomyocytes and its consequences for $\mathrm{Ca}^{2+}$ cycling is subject to intense debate. While it is well accepted that oscillations in mitochondrial $\mathrm{Ca}^{2+}$ occur during cytosolic $\mathrm{Ca}^{2+}$ transients (Boyman et al., 2014), there is conflicting evidence as to whether mitochondria play an active or passive role in calcium cycling, be that through changes in ATP production (Luongo et al., 2015), $\mathrm{Ca}^{2+}$ release (Zhao et al., 2013) or buffering of cytosolic $\mathrm{Ca}^{2+}$ transients (Drago et al., 2012; Boyman et al., 2014). A number of excellent reviews have been written on the subject (Lukyanenko et al., 2009; Kohlhaas and Maack, 2013).

In hiPSC-CMs, exposure to the mitochondrial uncoupler FCCP, at low concentrations reported to enhance mitochondrial oxidative respiration without changing membrane potential (Brennan et al., 2006), abolished the occurrence of spontaneous $\mathrm{Ca}^{2+}$ transients (Zhang et al., 2015). This stands in stark contrast to the finding in mouse ventricular cardiomyocytes that identical concentrations of FCCP enhance spontaneous SR release by releasing $\mathrm{Ca}^{2+}$ from mitochondria (Zhao et al., 2013). The ability of mitochondria to affect SR release is suggested to be due to the formation of functional micro-domains between the two structures, where small movements of $\mathrm{Ca}^{2+}$ can produce large changes in local concentration (Lu et al., 2013). The limited ultrastructural organization of hiPSC-CMs may mean that these micro-domains are lost.

However, in the same study, the genetically encoded mitochondrial $\mathrm{Ca}^{2+}$ sensing probe mitycam-E31Q revealed that, during spontaneous depolarization, mitochondrial $\mathrm{Ca}^{2+}$ dynamics displayed regional variability, with the perinuclear mitochondrial population releasing $\mathrm{Ca}^{2+}$ while peripheral organelles sequestered $\mathrm{Ca}^{2+}$ (Zhang et al., 2015). While the authors do not provide evidence as to whether this mitochondrial $\mathrm{Ca}^{2+}$ movement precedes or is a consequence of $\mathrm{SR} \mathrm{Ca}^{2+}$ release, the correlation between mitochondrial $\mathrm{Ca}^{2+}$ release, cellular micro-domains prone to spontaneous $\mathrm{Ca}^{2+}$ release events and the perinuclear generation of whole cell $\mathrm{Ca}^{2+}$ transients suggests mitochondrial activity plays a role in regulating $\mathrm{Ca}^{2+}$ cycling in these cells.

\section{Structure-function Relationships in hiPSC-CMs}

The lack of the sophisticated adult myocyte structure in hiPSCCMs may explain some functional observations. From studies discussed here it is clear that $\mathrm{SR} \mathrm{Ca}^{2+}$ release is limited in $\mathrm{Ca}^{2+}$-induced transients, with fractional release, calculated as $\Delta \mathrm{F}_{(I C a)} / \Delta \mathrm{F}_{(\text {Caff })}$, averaging at $\sim 0.3$ vs. $0.6-0.9$ in adult human cells (Bassani et al., 1995; Zhang et al., 2013b). Inhomogeneous clustering of RyRs, the consequent loss of local control of $\mathrm{Ca}^{2+}$ release and limited association with sarcolemmal $\mathrm{Ca}^{2+}$ channels contributes to slow and inefficient rise of $\left[\mathrm{Ca}^{2+}\right]_{i}$. hiPSC-CMs are devoid of t-tubules (Gherghiceanu et al., 2011), the sites of dyad formation in the cardiomyocyte. The absence of t-tubules in adult cells, in atrial or failing cardiomyocytes for example, correlates with a number of properties we have discussed here in hiPSC-CMs such as sub-sarcolemmal generation of $\mathrm{Ca}^{2+}$ transients, low fractional release and increased dependence on sarcolemmal $\mathrm{Ca}^{2+}$ flux (Figure 1). This structural immaturity may be a limiting step in these cells becoming more functionally mature.

Equally so the opposite can be argued. T-tubule formation and maintenance is an inherently energy-intensive process. Low SERCA expression and $\mathrm{Ca}^{2+}$ extrusion rate, high PLN:SERCA ratio and a negative force-frequency response point to decreased SR function and regulation, associated with a greater dependence on sarcolemmal flux to source and extrude $\mathrm{Ca}^{2+}$. As such, greater structural co-ordination may yield limited benefits without improvements in the underlying function of EC coupling components, and vice-versa.

Standard culture environments place little demand on hiPSCCMs in terms of $\mathrm{Ca}^{2+}$ handling, and as such it could be argued that it is more efficient to rely on sarcolemmal flux driven by electrochemical gradients than ATP-intensive processes. We and others have shown how using culture techniques to manipulate myocyte structure can result in substantial changes in $\mathrm{Ca}^{2+}$ 
handling properties (Kaji et al., 2003; Yin, 2004; Rao et al., 2013; Trantidou et al., 2014).

\section{Conclusion and Future Directions}

hiPSC-CMs hold enormous promise in many fields of cardiovascular research. While we have a reasonable understanding of the $\mathrm{Ca}^{2+}$ handling properties of these cells a number of questions remain, particularly with regards to how well $\mathrm{Ca}^{2+}$ channels and RyRs are coupled and what the dominant sources of $\mathrm{Ca}^{2+}$ are in EC coupling.

With many of the end applications of hiPSC-CMs dependent on the ability of these cells to recapitulate adult human cardiomyocyte properties to a satisfactory degree, differences in channel pharmacology, mechanisms of automaticity and structural organization between hiPSC-CMs and adult cardiomyocytes pose challenges to fully realizing the potential of this technology. It is important however to remember that there is no truly definitive understanding of human cardiac physiology. Difficulties in obtaining healthy adult tissue have meant that the field has come to rely on failing hearts, and in particular on rabbit physiology, as a basis of comparison. While this by no means invalidates the comparative studies above nor the concerns raised about hiPSC-CM function, it is essential to consider this limitation when discussing hiPSC-CM properties.

\section{References}

Ai, X., Curran, J. W., Shannon, T. R., Bers, D. M., and Pogwizd, S. M. (2005). Ca2+/calmodulin-dependent protein kinase modulates cardiac ryanodine receptor phosphorylation and sarcoplasmic reticulum $\mathrm{Ca} 2+$ leak in heart failure. Circ. Res. 97, 1314-1322. doi: 10.1161/01.RES.0000194329. 41863.89

Bassani, J. W., Yuan, W., and Bers, D. M. (1995). Fractional SR Ca release is regulated by trigger $\mathrm{Ca}$ and SR Ca content in cardiac myocytes. Am. J. Physiol. 268, C1313-C1319.

Bellin, M., Marchetto, M. C., Gage, F. H., and Mummery, C. L. (2012). Induced pluripotent stem cells: the new patient? Nat. Rev. Mol. Cell Biol. 13, 713-726. doi: $10.1038 / \mathrm{nrm} 3448$

Bers, D. M. (2002). Excitation-Contraction Coupling and Cardiac Contractile Force. Dordrecht: Kluwer Academic Publishers.

Blaustein, M. P., and Lederer, W. J. (1999). Sodium/calcium exchange: its physiological implications. Physiol. Rev. 79, 763-854.

Blazeski, A., Zhu, R., Hunter, D. W., Weinberg, S. H., Zambidis, E. T., and Tung, L. (2012). Cardiomyocytes derived from human induced pluripotent stem cells as models for normal and diseased cardiac electrophysiology and contractility. Prog. Biophys. Mol. Biol. 110, 166-177. doi: 10.1016/j.pbiomolbio.2012. 07.013

Boyman, L., Chikando, A. C., Williams, G. S. B., Khairallah, R. J., Kettlewell, S., Ward, C. W., et al. (2014). Calcium movement in cardiac mitochondria. Biophys. J. 107, 1289-1301. doi: 10.1016/j.bpj.2014.07.045

Brennan, J. P., Berry, R. G., Baghai, M., Duchen, M. R., and Shattock, M. J. (2006). FCCP is cardioprotective at concentrations that cause mitochondrial oxidation without detectable depolarisation. Cardiovasc. Res. 72, 322-330. doi: 10.1016/j.cardiores.2006.08.006

Caspi, O., Huber, I., Gepstein, A., Arbel, G., Maizels, L., Boulos, M., et al. (2013). Modeling of arrhythmogenic right ventricular cardiomyopathy with human induced pluripotent stem cells. Circ. Cardiovasc. Genet. 6, 557-568. doi: 10.1161/CIRCGENETICS.113.000188
From a more practical perspective, the minimal variability shown between different cells lines and different differentiation protocols is reassuring, and gives us greater confidence when comparing and collating the work of multiple groups. Consideration must be given however to culture conditions, with substantial variability in hiPSC-CM electrophysiology apparently dependent on cell density (Du et al., 2015). Further work will be needed to determine the general applicability of studies that necessitate the use of single cells and to develop more suitable alternatives.

Overall, a more solid understanding of the electrophysiological properties and limits of hiPSC-CMs will be extremely valuable in the interpretation of the effects of novel drugs and making judgments on the ability of these cells to successfully recapitulate human disease.

\section{Authorship}

We confirm that CK, LC, and CT meet the criteria for authorship of this review based on the recommendations of the ICMJE.

\section{Funding}

$\mathrm{CK}$ is funded by a British Heart Foundation $\mathrm{MBBS} / \mathrm{PhD}$ studentship FS/13/46/30282.

Chang, M. G., Tung, L., Sekar, R. B., Chang, C. Y., Cysyk, J., Dong, P., et al. (2006). Proarrhythmic potential of mesenchymal stem cell transplantation revealed in an in vitro coculture model. Circulation 113, 1832-1841. doi: 10.1161/CIRCULATIONAHA.105.593038

Chen, H.-S. V., Kim, C., and Mercola, M. (2009). Electrophysiological challenges of cell-based myocardial repair. Circulation 120, 2496-2508. doi: 10.1161/CIRCULATIONAHA.107.751412

Chen, X., Zhang, X., Harris, D. M., Piacentino, V., Berretta, R. M., Margulies, K. B., et al. (2008). Reduced effects of BAY K 8644 on L-type Ca2+ current in failing human cardiac myocytes are related to abnormal adrenergic regulation. Am. J. Physiol. Heart Circ. Physiol. 294, H2257-H2267. doi: 10.1152/ajpheart.01335.2007

Di Pasquale, E., Lodola, F., Miragoli, M., Denegri, M., Avelino-Cruz, J. E., Buonocore, M., et al. (2013). CaMKII inhibition rectifies arrhythmic phenotype in a patient-specific model of catecholaminergic polymorphic ventricular tachycardia. Cell Death Dis. 4, e843. doi: 10.1038/cddis.2013.369

Drago, I., De Stefani, D., Rizzuto, R., and Pozzan, T. (2012). Mitochondrial Ca2+ uptake contributes to buffering cytoplasmic Ca2+ peaks in cardiomyocytes. Proc. Natl. Acad. Sci. U.S.A. 109, 12986-12991. doi: 10.1073/pnas.1210718109

Du, D. T. M., Hellen, N., Kane, C., and Terracciano, C. M. N. (2015). Biophysical letter action potential morphology of human induced pluripotent stem cellderived cardiomyocytes does not predict cardiac chamber specificity and is dependent on cell density. Biophys. J. 108, 1-4. doi: 10.1016/j.bpj.2014.11.008

Fatima, A., Xu, G., Shao, K., Papadopoulos, S., Lehmann, M., Arnáiz-Cot, J. J., et al. (2011). In vitro modeling of ryanodine receptor 2 dysfunction using human induced pluripotent stem cells. Cell. Physiol. Biochem. 28, 579-592. doi: $10.1159 / 000335753$

Fine, M., Lu, F.-M., Lin, M.-J., Moe, O., Wang, H.-R., and Hilgemann, D. W. (2013). Human-induced pluripotent stem cell-derived cardiomyocytes for studies of cardiac ion transporters. Am. J. Physiol. Cell Physiol. 305, C481-C491. doi: 10.1152/ajpcell.00143.2013

Germanguz, I., Sedan, O., Zeevi-Levin, N., Shtrichman, R., Barak, E., Ziskind, A., et al. (2011). Molecular characterization and functional properties of 
cardiomyocytes derived from human inducible pluripotent stem cells. J. Cell. Mol. Med. 15, 38-51. doi: 10.1111/j.1582-4934.2009.00996.x

Gherghiceanu, M., Barad, L., Novak, A., Reiter, I., Itskovitz-Eldor, J., Binah, O., et al. (2011). Cardiomyocytes derived from human embryonic and induced pluripotent stem cells: comparative ultrastructure. J. Cell. Mol. Med. 15, 2539-2551. doi: 10.1111/j.1582-4934.2011.01417.x

Go, L. O., Moschella, M. C., Watras, J., Handa, K. K., Fyfe, B. S., and Marks, A. R. (1995). Differential regulation of two types of intracellular calcium release channels during end-stage heart failure. J. Clin. Invest. 95, 888-894. doi: 10.1172/JCI117739

Hwang, H. S., Kryshtal, D. O., Feaster, T. K., Sánchez-Freire, V., Zhang, J., Kamp, T. J., et al. (2015). Comparable calcium handling of human iPSC-derived cardiomyocytes generated by multiple laboratories. J. Mol. Cell. Cardiol. 85, 79-88. doi: 10.1016/j.yjmcc.2015.05.003

Itzhaki, I., Rapoport, S., Huber, I., Mizrahi, I., Zwi-Dantsis, L., Arbel, G., et al. (2011). Calcium handling in human induced pluripotent stem cell derived cardiomyocytes. PLoS ONE 6:e18037. doi: 10.1371/journal.pone.0018037

Ji, J., Kang, J., and Rampe, D. (2014). L-type Ca2+ channel responses to bay $\mathrm{k}$ 8644 in stem cell-derived cardiomyocytes are unusually dependent on holding potential and charge carrier. Assay Drug Dev. Technol. 12, 352-360. doi: 10.1089/adt.2014.596

Jonsson, M. K. B., Vos, M. A., Mirams, G. R., Duker, G., Sartipy, P., de Boer, T. P., et al. (2012). Application of human stem cell-derived cardiomyocytes in safety pharmacology requires caution beyond hERG. J. Mol. Cell. Cardiol. 52, 998-1008. doi: 10.1016/j.yjmcc.2012.02.002

Kaji, H., Takoh, K., Nishizawa, M., and Matsue, T. (2003). Intracellular Ca2+ imaging for micropatterned cardiac myocytes. Biotechnol. Bioeng. 81, 748-751. doi: 10.1002/bit.10521

Kang, J., Chen, X.-L., Ji, J., Lei, Q., and Rampe, D. (2012). $\mathrm{Ca}^{2+}$ channel activators reveal differential L-type $\mathrm{Ca}^{2+}$ channel pharmacology between native and stem cell-derived cardiomyocytes. J. Pharmacol. Exp. Ther. 341, 510-517. doi: 10.1124/jpet.112.192609

Kapur, N., and Banach, K. (2007). Inositol-1,4,5-trisphosphate-mediated spontaneous activity in mouse embryonic stem cell-derived cardiomyocytes. J. Physiol. 581, 1113-1127. doi: 10.1113/jphysiol.2006.125955

Khan, J. M., Lyon, A. R., and Harding, S. E. (2013). The case for induced pluripotent stem cell-derived cardiomyocytes in pharmacological screening. $B r$. J. Pharmacol. 169, 304-317. doi: 10.1111/j.1476-5381.2012.02118.x

Kim, J. J., Yang, L., Lin, B., Zhu, X., Sun, B., Kaplan, A. D., et al. (2015). Mechanism of automaticity in cardiomyocytes derived from human induced pluripotent stem cells. J. Mol. Cell. Cardiol. 81, 81-93. doi: 10.1016/j.yjmcc.2015.01.013

Kohlhaas, M., and Maack, C. (2013). Calcium release microdomains and mitochondria. Cardiovasc. Res. 98, 259-268. doi: 10.1093/cvr/cvt032

Kola, I., and Landis, J. (2004). Can the pharmaceutical industry reduce attrition rates? Nat. Rev. Drug Discov. 3, 711-715. doi: 10.1038/nrd1470

Lalit, P. A., Hei, D. J., Raval, A. N., and Kamp, T. J. (2014). Induced pluripotent stem cells for post-myocardial infarction repair: remarkable opportunities and challenges. Circ. Res. 114, 1328-1345. doi: 10.1161/CIRCRESAHA.114. 300556

Lee, Y. K., Ng, K. M., Lai, W. H., Chan, Y. C., Lau, Y. M., Lian, Q., et al. (2011). Calcium homeostasis in human induced pluripotent stem cell-derived cardiomyocytes. Stem Cell Rev. 7, 976-986. doi: 10.1007/s12015-011-9273-3

Lin, B., Li, Y., Han, L., Kaplan, A. D., Ao, Y., Kalra, S., et al. (2015). Modeling and study of the mechanism of dilated cardiomyopathy using induced pluripotent stem cells derived from individuals with Duchenne muscular dystrophy. Dis. Model. Mech. 8, 457-466. doi: 10.1242/dmm.019505

Liu, Q.-N., and Trudeau, M. C. (2015). Eag domains regulate LQT mutant hERG channels in human induced pluripotent stem cell-derived cardiomyocytes. PLoS ONE 10:e0123951. doi: 10.1371/journal.pone.0123951

Lu, X., Ginsburg, K. S., Kettlewell, S., Bossuyt, J., Smith, G. L., and Bers, D. M. (2013). Measuring local gradients of intramitochondrial $[\mathrm{Ca}(2+)]$ in cardiac myocytes during sarcoplasmic reticulum $\mathrm{Ca}(2+)$ release. Circ. Res. 112, 424-431. doi: 10.1161/CIRCRESAHA.111.300501

Lukyanenko, V., Chikando, A., and Lederer, W. J. (2009). Mitochondria in cardiomyocyte Ca2+ signaling. Int. J. Biochem. Cell Biol. 41, 1957-1971. doi: 10.1016/j.biocel.2009.03.011

Luongo, T. S., Lambert, J. P., Yuan, A., Zhang, X., Gross, P., Song, J., et al. (2015). The mitochondrial calcium uniporter matches energetic supply with cardiac workload during stress and modulates permeability transition. Cell Rep. 12, 23-34. doi: 10.1016/j.celrep.2015.06.017

Ma, D., Wei, H., Lu, J., Huang, D., Liu, Z., Loh, L. J., et al. (2015). Characterization of a novel KCNQ1 mutation for type 1 long QT syndrome and assessment of the therapeutic potential of a novel IKs activator using patient-specific induced pluripotent stem cell-derived cardiomyocytes. Stem Cell Res. Ther. 6, 39. doi: 10.1186/s13287-015-0027-z

Ma, J., Guo, L., Fiene, S. J., Anson, B. D., Thomson, J. A., Kamp, T. J., et al. (2011). High purity human-induced pluripotent stem cell-derived cardiomyocytes: electrophysiological properties of action potentials and ionic currents. Am. J. Physiol. Circ. Physiol. 301, H2006-H2017. doi: 10.1152/ajpheart.00694.2011

Macmillan, D., and McCarron, J. G. (2010). The phospholipase C inhibitor U73122 inhibits $\mathrm{Ca}(2+)$ release from the intracellular sarcoplasmic reticulum $\mathrm{Ca}(2+)$ store by inhibiting $\mathrm{Ca}(2+)$ pumps in smooth muscle. Br. J. Pharmacol. 160, 1295-1301. doi: 10.1111/j.1476-5381.2010.00771.x

Mauritz, C., Schwanke, K., Reppel, M., Neef, S., Katsirntaki, K., Maier, L. S., et al. (2008). Generation of functional murine cardiac myocytes from induced pluripotent stem cells. Circulation 118, 507-517. doi: 10.1161/CIRCULATIONAHA.108.778795

Mullard, A. (2014). New drugs cost US $\$ 2.6$ billion to develop. Nat. Rev. Drug Discov. 13, 877-877. doi: 10.1038/nrd4507

Narazaki, G., Uosaki, H., Teranishi, M., Okita, K., Kim, B., Matsuoka, S., et al. (2008). Directed and systematic differentiation of cardiovascular cells from mouse induced pluripotent stem cells. Circulation 118, 498-506. doi: 10.1161/CIRCULATIONAHA.108.769562

Novak, A., Lorber, A., Itskovitz-Eldor, J., and Binah, O. (2012). Modeling catecholaminergic polymorphic ventricular tachycardia using induced pluripotent stem cell-derived cardiomyocytes. Rambam Maimonides Med. J. 3, e0015. doi: 10.5041/RMMJ.10086

Paul, S. M., Mytelka, D. S., Dunwiddie, C. T., Persinger, C. C., Munos, B. H., Lindborg, S. R., et al. (2010). How to improve R\&D productivity: the pharmaceutical industry's grand challenge. Nat. Rev. Drug Discov. 9, 203-214. doi: $10.1038 / \operatorname{nrd} 3078$

Poindexter, B. J., Smith, J. R., Buja, L. M., and Bick, R. J. (2001). Calcium signaling mechanisms in dedifferentiated cardiac myocytes: comparison with neonatal and adult cardiomyocytes. Cell Calcium 30, 373-382. doi: 10.1054/ceca.2001.0249

Pott, C., Eckardt, L., and Goldhaber, J. I. (2011). Triple threat: the Na+/Ca2+ exchanger in the pathophysiology of cardiac arrhythmia, ischemia and heart failure. Curr. Drug Targets 12, 737-747. doi: 10.2174/138945011795378559

Rajamohan, D., Matsa, E., Kalra, S., Crutchley, J., Patel, A., George, V., et al. (2013). Current status of drug screening and disease modelling in human pluripotent stem cells. Bioessays 35, 281-298. doi: 10.1002/bies.201200053

Rao, C., Prodromakis, T., Kolker, L., Chaudhry, U. A., Trantidou, T., Sridhar, A., et al. (2013). The effect of microgrooved culture substrates on calcium cycling of cardiac myocytes derived from human induced pluripotent stem cells. Biomaterials 34, 2399-2411. doi: 10.1016/j.biomaterials.2012.11.055

Sartiani, L., Bettiol, E., Stillitano, F., Mugelli, A., Cerbai, E., and Jaconi, M. E. (2007). Developmental changes in cardiomyocytes differentiated from human embryonic stem cells: a molecular and electrophysiological approach. Stem Cells 25, 1136-1144. doi: 10.1634/stemcells.2006-0466

Sharma, A., Wu, J. C., and Wu, S. M. (2013). Induced pluripotent stem cell-derived cardiomyocytes for cardiovascular disease modeling and drug screening. Stem Cell Res. Ther. 4, 150. doi: 10.1186/scrt380

Sinnecker, D., Laugwitz, K.-L., and Moretti, A. (2014). Induced pluripotent stem cell-derived cardiomyocytes for drug development and toxicity testing. Pharmacol. Ther. 143, 246-252. doi: 10.1016/j.pharmthera.2014.03.004

Smit, N. W., and Coronel, R. (2014). Stem cells can form gap junctions with cardiac myocytes and exert pro-arrhythmic effects. Front. Physiol. 5:419. doi: 10.3389/fphys.2014.00419

Stevens, J. L., and Baker, T. K. (2009). The future of drug safety testing: expanding the view and narrowing the focus. Drug Discov. Today 14, 162-167. doi: 10.1016/j.drudis.2008.11.009

Takahashi, K., and Yamanaka, S. (2006). Induction of pluripotent stem cells from mouse embryonic and adult fibroblast cultures by defined factors. Cell 126, 663-676. doi: 10.1016/j.cell.2006.07.024

Trantidou, T., Rao, C., Barrett, H., Camelliti, P., Pinto, K., Yacoub, M. H., et al. (2014). Selective hydrophilic modification of Parylene C films: a new approach 
Kane et al.

EC Coupling of hiPSC-CMs

to cell micro-patterning for synthetic biology applications. Biofabrication 6, 025004. doe: 10.1088/1758-5082/6/2/025004

Wen, J.-Y., Wei, C.-Y., Shah, K., Wong, J., Wang, C., and Chen, H.-S. V. (2015). Maturation-based model of arrhythmogenic right ventricular dysplasia using patient-specific induced pluripotent Stem Cells. Circe. J. 79, 1462-1468. doi: 10.1253/circj.CJ-15-0363

Yin, L. (2004). Scaffold topography alters intracellular calcium dynamics in cultured cardiomyocyte networks. AJP Hear. Circe. Physiol. 287, H1276-H1285. doa: 10.1152/ajpheart.01120.2003

Yokoo, N., Baba, S., Kaichi, S., Niwa, A., Mira, T., Dor, H., et al. (2009). The effects of cardioactive drugs on cardiomyocytes derived from human induced pluripotent stem cells. Biochem. Biophys. Res. Commune. 387, 482-488. do: 10.1016/j.bbrc.2009.07.052

Chang, G. Q., Wei, H., Lu, J., Wong, P., and Shim, W. (2013a). Identification and characterization of calcium sparks in cardiomyocytes derived from human induced pluripotent stem cells. PLOS ONE 8:e55266. dor: 10.1371/journal.pone.0055266

Chang, J., Wilson, G. F., Soerens, A. G., Koonce, C. H., Yu, J., Palecek, S. P., et al. (2009). Functional cardiomyocytes derived from human induced pluripotent stem cells. Circe. Res. 104, e30-e41. do: 10.1161/CIRCRESAHA.108. 192237

Chang, X.-H., Haviland, S., Wei, H., Saric, T., Fatima, A., Hescheler, J., et al. (2013b). Ca+ signaling in human induced pluripotent stem cell-derived cardiomyocytes (iPS-CM) from normal and catecholaminergic polymorphic ventricular tachycardia (CPVT)-afflicted subjects. Cell Calcium 54, 57-70. doa: 10.1016/j.ceca.2013.04.004

Chang, X.-H., Wei, H., Šaric, T., Hescheler, J., Cleemann, L., and Monad, M. (2015). Regionally diverse mitochondrial calcium signaling regulates spontaneous pacing in developing cardiomyocytes. Cell Calcium 57, 321-336. dor: $10.1016 /$ j.ceca.2015.02.003

Chang, Y. M. (2002). Stem cell-derived cardiomyocytes demonstrate arrhythmic potential. Circulation 106, 1294-1299. doa: 10.1161/01.CIR.0000027585.05868.67

Zhao, Z., Gordan, R., Wen, H., Fefelova, N., Zany, W.-J., and Xis, L.-H. (2013). Modulation of intracellular calcium waves and triggered activities by mitochondrial ca flux in mouse cardiomyocytes. PLo ONE 8:e80574. doa: 10.1371/journal.pone.0080574

Conflict of Interest Statement: The authors declare that the research was conducted in the absence of any commercial or financial relationships that could be construed as a potential conflict of interest.

Copyright (c) 2015 Kane, Couch and Terracciano. This is an open-access article distributed under the terms of the Creative Commons Attribution License (CC BY). The use, distribution or reproduction in other forums is permitted, provided the original authors) or licenser are credited and that the original publication in this journal is cited, in accordance with accepted academic practice. No use, distribution or reproduction is permitted which does not comply with these terms.

Frontiers in Cell and Developmental Biology | www.frontiersin.org

8

September 2015 | Volume 3 | Article 59 